\title{
Genomic rearrangements in childhood spinal muscular atrophy: linkage disequilibrium with a null allele
}

Rachael J Daniels, Louise Campbell, Nanda R Rodrigues, Michael J Francis, Karen E Morrison, Michael McLean, Alex MacKenzie, Jaakko Ignatius, Victor Dubowitz, Kay E Davies

\begin{abstract}
Autosomal recessive childhood onset spinal muscular atrophy has been mapped to chromosome 5q13. We report the analysis of a polymorphic microsatellite which shows linkage disequilibrium with the disease. The linkage disequilibrium is observed with a null allele which is seen as the non-inheritance of alleles from one or both parents. The inheritance of a null allele was observed in 26 out of $36(72 \%)$ informative childhood onset spinal muscular atrophy (SMA) families tested, of all types of severity and from a variety of ethnic backgrounds. In seven families segregating for the severe WerdnigHoffmann or SMA type I, no alleles were inherited from either parent using this microsatellite. This null allele may represent a deletion which is either closely associated with, or causes, the disease.
\end{abstract}

\section{( 7 Med Genet 1995;32:93-96)}

Childhood onset proximal spinal muscular atrophy (SMA) has been well defined clinically and genetically. ${ }^{1-8}$ It is a common autosomal recessive neurological disorder in which the initial biochemical defect is unknown, which results in abnormalities of the alpha motor neurones in the anterior horn of the spinal cord. Molecular genetic research has localised the mutation(s) to chromosome 5q11.2q13.3. ${ }^{9-13}$ Genetic linkage analysis and the consideration of key recombinant family data has mapped the gene to the interval between the markers D5S435 and D5S557..$^{9-11} 13$ Yeast artificial chromosome (YAC) contigs of this region have been constructed and new microsatellites reported..$^{13-16}$ The candidate region contains chromosome 5 specific repeat sequences as evidenced by the detailed study of genomic and cDNA sequences that map within it. ${ }^{1317}$ A microsatellite repeat, CATT-1, isolated by Burges et al, ${ }^{16}$ has multiple sites within the region, with as many as eight alleles in any one person. In an attempt to distinguish between the individual sites for CATT-1, alternative primers flanking the various copies of this dinucleotide repeat were obtained. Genotyping with one set of these alternative primers (defining the locus CATT/alt) shows linkage disequilibrium with SMA.

Melki et al $^{15}$ recently published three microsatellites, C272, C212, and C161, which also have multiple copies in the candidate region and show abnormal genetic changes within a small proportion of SMA families. We have analysed these markers in our data set and compare them with the results obtained with CATT/alt.

\section{Materials and methods \\ FAMILIES}

A total of 130 SMA families was used in this study, 101 of which have been previously tested

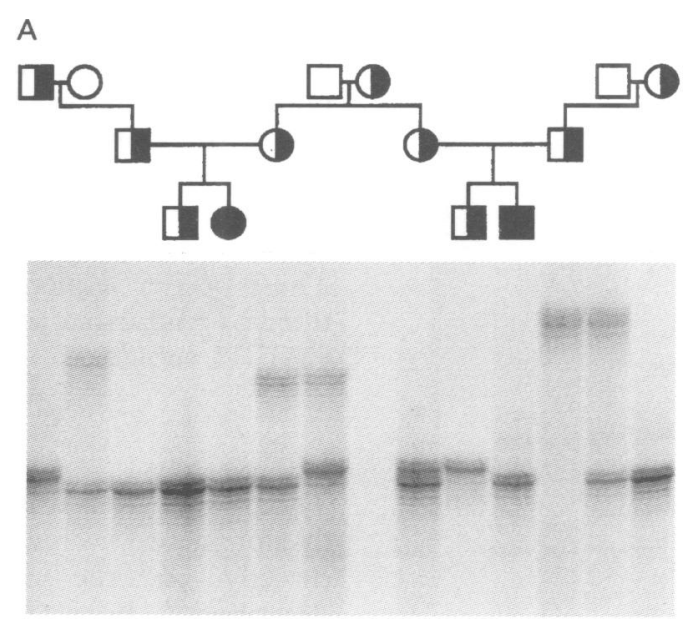

B
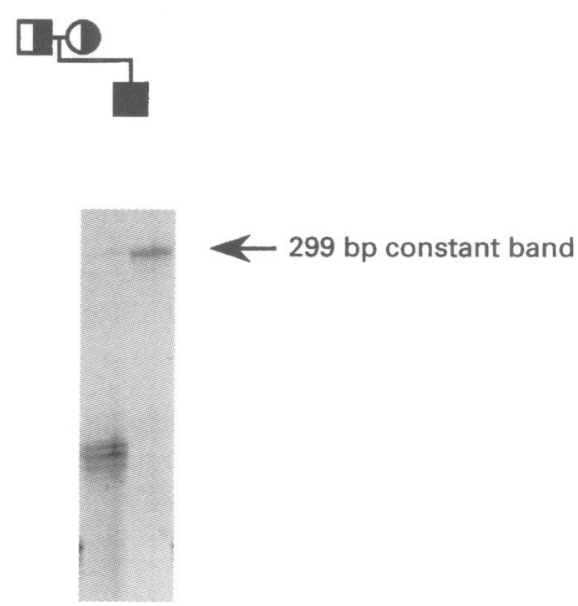

Figure 1(A) Example of the lack of inheritance of one parental allele at the CATT/alt locus in an SMA type family (UK). (B) Example of lack of inheritance of alleles from each parent at the CATT/alt locus in an SMA type I family (UK). The father's DNA was unavailable type I analysis. 
Table 1 Analysis of CATT/alt in SMA families and controls

\begin{tabular}{|c|c|c|c|}
\hline \multirow[b]{2}{*}{ Families } & \multicolumn{3}{|c|}{ Inheritance } \\
\hline & $\begin{array}{l}\text { No allele } \\
\text { absence }\end{array}$ & $\begin{array}{l}\text { Allele absence } \\
\text { from one } \\
\text { parent }\end{array}$ & $\begin{array}{l}\text { Allele absence } \\
\text { from both } \\
\text { parents }\end{array}$ \\
\hline Finnish I & 2 & 3 & 4 \\
\hline Finnish II & 3 & 6 & 0 \\
\hline Finnish III & 0 & 4 & 0 \\
\hline Other I & 5 & 6 & 3 \\
\hline Totals & 10 & 19 & 7 \\
\hline Non-SMA & 24 & 0 & 0 \\
\hline
\end{tabular}

I, II, and III refer to the types of SMA

"Other" includes families from the UK, elsewhere in Europe, and Asia.

Ten SMA families were uninformative, 6 non-SMA control families were uninformative.

with other markers within the region which show genetic linkage with SMA. The remainder represent families who have a single affected child or families for whom no haplotyping has yet been performed. All cases conform to the international criteria for diagnosis of SMA. ${ }^{2}$
A
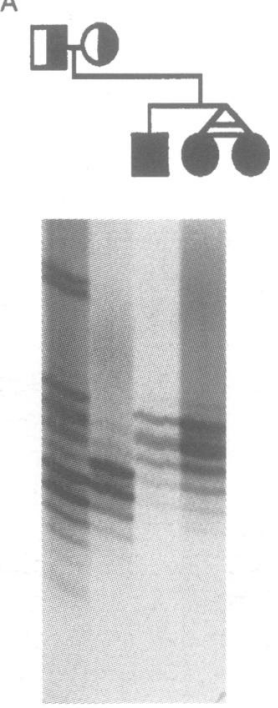

B
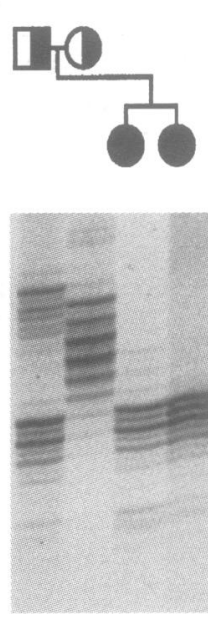

Figure 2(A) Example of the allele loss at the C272 locus in an SMA type I family (UK). (B)

Example of allele loss at the C272 locus in an SMA type III family (UK).
Table 2 Analysis of C272 in SMA families and controls

\begin{tabular}{lll}
\hline & \multicolumn{2}{l}{ Inheritance } \\
\cline { 2 - 3 } Families & $\begin{array}{l}\text { No allele } \\
\text { absence }\end{array}$ & $\begin{array}{l}\text { Allele absence from } \\
\text { one parent }\end{array}$ \\
\hline Finnish I & 2 & 0 \\
Finnish II & 7 & 0 \\
Finnish III & 4 & 0 \\
Other I & 4 & 1 \\
Other II & 12 & 0 \\
Other III & 2 & 1 \\
Totals & 31 & 2 \\
Non-SMA & 28 & 0
\end{tabular}

I, II, and III refer to the types of SMA.

"Other" includes families from the UK, elsewhere in Europe, and Asia.

Seventy-five SMA families were uninformative, 1 non-SMA control family was uninformative.

and Asia, with the microsatellite primers from the CATT/alt locus (see Methods). The results are summarised in table 1 . An example of a family for whom the affected child fails to inherit an allele from one of its parents is given in fig 1A. In the left half of the pedigree, the father of the affected child has not inherited any alleles from his father. The next mating is uninformative because both parents have the same allele. Similarly, on the far right of the pedigree, the carrier grandmother does not give an allele to her carrier son, who in turn does not pass on any visible allele to his affected son.

An example of an affected child who fails to inherit a visible allele from either parent is given in fig 1B. This is not the result of technical failure because the upper constant band at $299 \mathrm{bp}$ (marked) is present. This band appears in the majority of tracks, except the negative control. This child's DNA sample gave appropriate PCR products for all other closely linked markers from the region. For the families that are informative at this locus, $72 \%(26 / 36)$ show an absence of an allele indicating the segregation of a null allele or deletion. Thirty families not suffering from SMA were also studied, none of which showed any absence of alleles (24 were informative, see table 1 ).

We also analysed the microsatellites reported by Melki et al, ${ }^{15} \mathrm{C} 272, \mathrm{C} 212$, and $\mathrm{C} 161$. The C272 microsatellite was analysed in the same families as those studied at the CATT/alt locus. None of these families showed allele absence, nor did the 29 control families. Since the frequency of allele absence at these loci is low, ${ }^{15}$ we extended the study in a further $62 \mathrm{SMA}$ families. The results for $\mathrm{C} 272$ are summarised in table 2. In two SMA families (type I and type III), the affected sibs failed to inherit a product from the mother (fig $2 \mathrm{~A}$ and B). Neither of these families showed the allele absence at the CATT/alt locus, where they were informative.

The C212 microsatellite was also tested in 82 SMA families and 25 non-SMA families and showed no abnormalities in inheritance. The sequence of the $\mathrm{C} 161$ primers was compared to the sequence published for the CATT1 loci and found to be very similar. When $\mathrm{C} 161$ was genotyped in the same families previously analysed with the CATT-1 primers, the PCR pattern was found to be the same (data not

\section{FAMILY STUDIES}

We analysed 28 Finnish families segregating for all three types of SMA and 18 SMA type I families from Europe (predominantly the UK) 


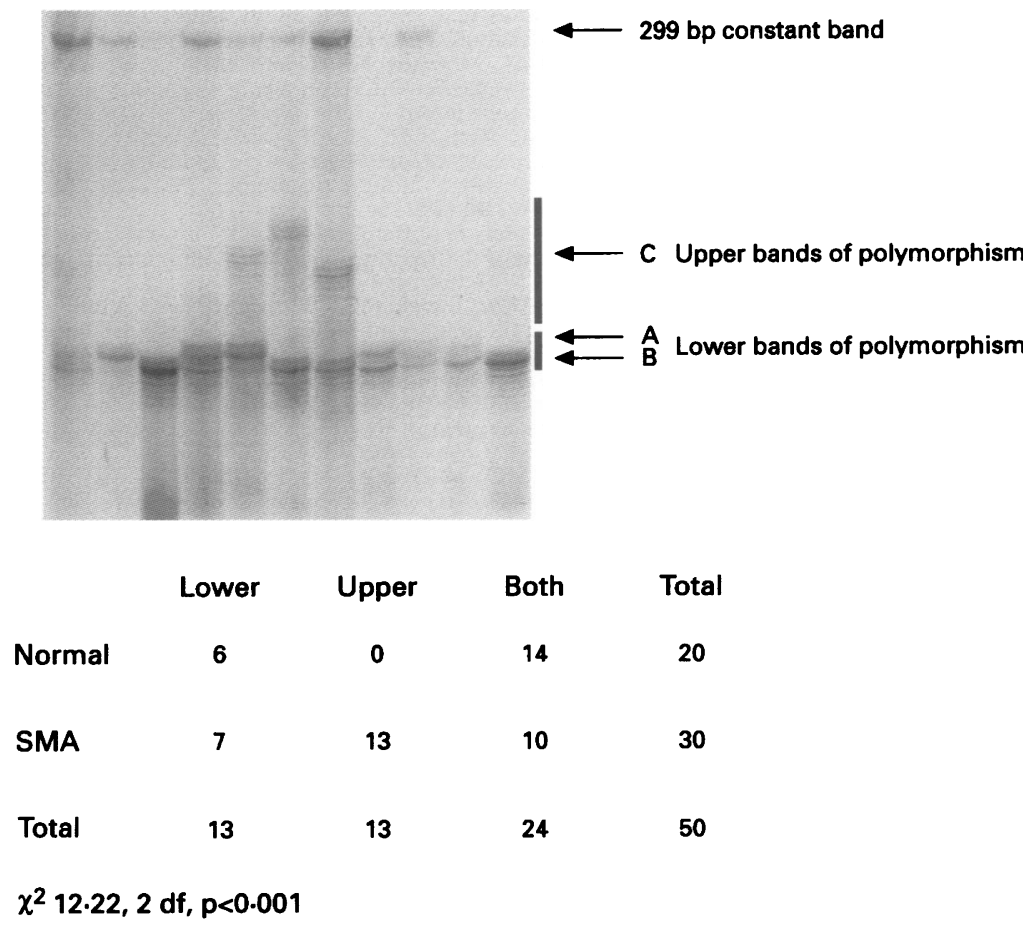

Figure 3 Linkage disequilibrium between SMA and the CATT/alt locus in the Finnish population. (fig 1A). The null allele was seen to be inherited from previous generations through both the maternal and paternal lines. When the genotypes from the other polymorphic loci in this region are studied, it is clear that the abnormality is exclusively inherited from carrier grandparents. None of the 30 non-SMA families studied showed an abnormality at the CATT/alt locus. The high numbers of SMA families showing allele absence suggests that this genetic change may have a direct link with the mutation involved in SMA.

Melki et $a l^{15}$ reported three families out of 201 (1.9\%) showing abnormalities at the C272 locus and three families (1.9\%) showing changes at the C212 locus. Dosage studies indicated that two of the families abnormal at the C212 locus represented a de novo change and one an inherited change, respectively. One further family showed a de novo rearrangement with both of these markers. In our studies, two out of 106 families $(1.8 \%)$ showed a similar genotypic change at the $\mathrm{C} 272$ locus, a result comparable to that found in the French cohort. Although we found no changes at the C212 locus, our data set may not be large enough to detect changes present at a frequency of 1 to $2 \%$. We found no evidence for de novo loss of alleles at the $\mathrm{C} 272$ locus as these changes were observed in more than one sib. Melki et $a l^{15}$ presented evidence for such de novo cases. If such de novo changes have occurred in our population, the parents must be germline mosaics.

In summary, we present in this paper the results of genotyping at a new locus, CATT/alt. This locus not only shows significant linkage disequilibrium with SMA in the Finnish population, implying that it may be very close to the gene, but the deletion/null allele present in at least half of the SMA families studied may relate to the mutation itself. As the families with null allele/deletions at the C272 locus are not the same as those which also show allele absence at the CATT/alt locus, the region over which rearrangements associated with the disease can occur may be quite large (several hundred kilobases). The seven SMA type I families showing no inheritance of visible alleles at the CATT/alt locus from both parents are worthy of further study as these might represent the extreme DNA rearrangement which results in the most severe phenotype.

\section{Discussion}

Seventy-two percent (26/36) of all informative SMA families showed abnormal inheritance patterns at the CATT/alt locus. This phenomenon was seen in families with all three forms of SMA, from the UK, Finland, Germany, and Asia. Similar results have recently also been described in Canadian and North American SMA type I patients. ${ }^{18}$ It is interesting to note that all the families which showed a "double null" inheritance pattern were of the severe SMA type I subgroup; this may in part explain the varying severity of the disease, if this "null allele" is in some way pathogenic. Furthermore, the three generation families available for study showed that the null allele was inherited from previous generations
We are very grateful to Dr Peter Harris for the DNA samples used as controls. We thank Helen Blaber for assistance with the preparation of the manuscript. We are indebted to the SMA families and the Finnish Red Cross for blood samples. We are grateful to the Medical Research Council, the Muscular Dystrophy Group of Great Britain and Northern Ireland, the Muscular Dystrophy Association in the USA, and the Finnish Muscular Dystrophy Association for generous support.

1 Dubowitz V. Muscle disorders in childhood. London: WB Saunders, 1978:146-78.

2 Munsat TL. Workshop Report: international SMA collaboration. Neuromusc Disord 1991;1:81.

3 Brzustowicz LM, Lehner T, Castilla LH, et al. Genetic mapping of chronic childhood-onset spinal muscular atrophy to chromosome 5q11.2-13.3. Nature 1990;344:5401.

4 Gilliam TC, Brzustowicz LM, Castilla LH, et al. Genetic homogeneity between acute and chronic forms of spinal muscular atrophy. Nature 1990;345:823-5.

5 Melki J, Abdelhak S, Sheth P, et al. Gene for chronic 5q. Nature 1990;344:767-8. proximal spinal muscular atrophies maps to chromosome 
6 Melki J, Sheth P, Abdelhak S, et al. Mapping of acute (type I) spinal muscular atrophy to chromosome 5q12-q14.

7 Daniels RJ, Thomas NH, MacKinnon RN, et al. Linkage analysis of spinal muscular atrophy. Genomics 1992;12: 335-9.

8 Morrison KE, Daniels RJ, Suthers GK, et al. High-resolution genetic map around the spinal muscular atrophy (SMA) locus on chromosome 5. Am f Hum Genet 1992;50:520-7. $9 \begin{aligned} & \text { Soares VM, Brzustowicz LM, Kleyn PW, et al. Refinement } \\ & \text { of the spinal muscular atrophy locus to the interval between }\end{aligned}$ D5S435 and MAP1B. Genomics 1993;15:365-71.

10 Wirth B, Pick E, Leutner A, et al. Large linkage analysis in 100 families with autosomal recessive spinal muscular atrophy (SMA) and 11 CEPH families using 15 polymorphic loci in the region 5q11.2-q13.3. Genomics 1994; 20:84-93.

11 Clermont O, Burlet P, Burglen L, et al. Use of genetic and physical mapping to locate the spinal muscular atrophy locus between two new highly polymorphic DNA markers. Am f Hum Genet 1994;54:687-94.

12 Brahe $\mathrm{C}$, Velonà I, van der Steege $\mathrm{G}$, et al. Mapping of two new markers within the smallest interval harboring the spinal muscular atrophy locus by family and radiation hybrid analysis. Hum Genet 1994;93:494-501.

13 Francis MJ, Morrison KE, Campbell L, et al. A contig of non-chimaeric YACs containing the spinal muscular atrophy gene in 5q13. Hum Mol Genet 1993;2:1161-7.

14 Kleyn PW, Wang CH, Lien LL, et al. Construction of a yeast artificial chromosome contig spanning the spina muscular atrophy disease gene region. Proc Natl Acad Sci USA 1993;90:6801-5.

15 Melki J, Lefebvre S, Burglen L, et al. De novo and inherited deletions of the 5 q13 region in spinal muscular atrophies. Science 1994;264:1474-7.

16 Burghes AHM, Ingraham SE, McLean M, et al. A multicopy dinucleotide marker that maps close to the spinal muscular atrophy gene. Genomics 1994;21:394-402.

17 Theodosiou AM, Morrison KE, Nesbit MA, et al. Complex repetitive arrangements of gene sequence in the refined repetitive arrangements of gene sequence in the refined
candidate region of the spinal muscular atrophy gene in candidate region of the spinal muscular a

18 McLean MD, MacKenzie AE, Roy N, et al. Two 5q13.1 microsatellite repeat loci are in linkage disequilibrium with type 1 spinal muscular atrophy. Hum Mol Genet 1994;3:1951-6. 